\title{
Concepciones de interculturalidad y práctica en aula: estudio con maestros de comunida- des shipibas en el Perú*
}

\author{
NORA CÉPEDA GARCÍA ${ }^{\text {雬 }}$ \\ Pontificia Universidad Católica del Perú \\ DINA CASTRO BURGOS \\ University of North Texas \\ PILAR LAMAS BASURTO \\ Pontificia Universidad Católica del Perú
}

Recibido el 18-10-2018; primera evaluación el 07-01-2019;

segunda evaluación el 10-12-2018; aceptado el 04-01-2019

\section{RESUMEN}

Este artículo presenta resultados de una investigación cualitativa y comparativa. Tuvo como objetivo general identificar las concepciones de interculturalidad de los docentes de dos escuelas interculturales bilingües shipibo-castellano en Lima y dos en Ucayali, y describir cómo están presentes en su práctica educativa de aula y planificación curricular. La metodología incluyó entrevistas semiestructuradas, observaciones y análisis de unidades de aprendizaje. Los resultados pasaron por

\footnotetext{
* Este estudio fue realizado con financiamiento de la Dirección de Gestión de la Investigación del Vicerrectorado de Investigación de la Pontificia Universidad Católica del Perú. La participación de la doctora Dina Castro Burgos fue cofinanciada por el Velma E. Schmidt Endowed Chair in Early Childhood Education de la University of North Texas.

** Docente contratada en la Pontificia Universidad Católica del Perú. Departamento Académico de Educación. Licenciada en Educación con especialidad en Educación Primaria, Segunda Especialidad en Formación Docente, maestría en Antropología. Experiencia en educación primaria, en formación docente inicial y en servicio, en elaboración de propuesta pedagógica y materiales educativos para niños y docentes, investigación en temas de docencia, en Tarea Asociación de Publicaciones Educativas. Correo: ncepeda@tarea.pe

*** Profesora principal en la University of North Texas (UNT), con un doctorado (Ph.D.) en Educación Infantil y Educación Especial (University of North Carolina in Chapel Hill), maestría en Salud Pública (University of Alabama in Birmingham), licenciatura en Psicología (Universidad Peruana Cayetano Heredia) y un certificado de posgrado en Estudios Latinoamericanos. Investigadora y evaluadora de proyectos sobre desarrollo bilingüe e interculturalidad en la educación infantil, con énfasis en acceso, calidad, formación y capacitación de maestros. Correo: dina.castro@unt.edu

**** Profesora ordinaria asociada de tiempo completo en el Departamento de Educación de la Pontificia Universidad Católica del Perú. Licenciada en Educación Inicial. Maestría en Educación con mención en Currículo. Profesora de Pre grado y Post Grado. Investigadora en temas curriculares. Correo: plamas@pucp.edu.pe
} 
un proceso de reducción de datos y triangulación. En la concepción de interculturalidad de los maestros se encontraron temáticas prevalentes: afirmación de la cultura propia, encuentro con otras lenguas y culturas e intercambio entre culturas. Se encontró relación entre sus concepciones y sus prácticas en aula. Mientras que la planificación curricular muestra diferencias en la incorporación de contenidos con perspectiva intercultural entre las escuelas de Lima y Ucayali.

Palabras clave: concepciones, educación intercultural, práctica docente, planificación curricular.

\section{Conceptions of Interculturality and classroom practice: A Study with Teachers of Shipibo Communities in Perú}

\section{Abstract}

This article presents the results from a qualitative and comparative study. The general objective was to identify the conceptions of interculturality of teachers in two intercultural bilingual Shipibo-Spanish schools in Lima and Ucayali, and describe how they are incorporated in their teaching practices and curricular planning. The methodology included a semi-structured interview, classroom observations, and the analysis of learning units. Findings resulted from a process of data reduction and triangulation. Prevalent themes were: the affirmation of their own culture, the encounter with other languages and cultures, and the exchange among cultures. There was a relation between teachers' conceptions and their classroom practices. Regarding curricular planning, there were differences in the incorporation of contents with an intercultural perspective between the schools in Lima and Ucayali.

Keywords: conceptions, intercultural education, teaching practice, curricular planning.

\section{Concepçóes de interculturalidade e prática em sala de aula: estudo com professores de comunidades Shipibas no Peru}

\section{Resumo}

$\mathrm{O}$ artigo apresenta resultados de pesquisa qualitativa e comparativa. Ele teve como objetivo geral identificar concepções interculturais dos professores em duas escolas interculturais bilíngües Shipibo-Castelhano em Lima e Ucayali e descrever como eles estão presentes em seu planejamento prática em sala de aula e currículo educacional. A metodologia incluiu a entrevista semiestruturada, observaçóes e análise de unidades de aprendizagem. Os resultados passaram por um processo de redução de dados e triangulação. $\mathrm{Na}$ concepçáo de interculturalidade dos professores, os temas predominantes foram encontrados: afirmação da própria cultura, encontro entre línguas e culturas, e troca entre culturas. Encontrou-se uma relação entre suas concepçóes e suas práticas de sala de aula. Enquanto o planejamento curricular mostra diferenças na incorporação de conteúdos com perspectiva intercultural entre as escolas de Lima e Ucayali.

Palavras-chave: Concepçóes, educação intercultural, prática de ensino, planejamento curricular. 


\section{INTRODUCCIÓN}

El Perú es un país de gran diversidad cultural, étnica, lingüística, geográfica y ecológica, alberga numerosos pueblos originarios andinos y amazónicos, además de mestizos, afrodescendientes e inmigrantes. El Ministerio de Cultura (2016) señala que los pueblos originarios suman 55 en el territorio nacional, donde se hablan 47 lenguas distintas al castellano y anteriores a los procesos de colonización europea de América. Uno de estos pueblos es el shipibo, uno de los más numerosos de la Amazonía peruana (Instituto Nacional de Estadística e Informática, 2009).

Durante el inicio de la república, las «formas de colonización interna estuvieron caracterizadas por la exclusión y denigración de lenguas, culturas y credos no ibéricos» (Valdiviezo y Valdiviezo, 2008). No es sino hasta 1972 que la Reforma Educativa del gobierno militar del general Juan Velasco Alvarado emite la primera Política Nacional de Educación Bilingüe destinada a los pueblos indígenas, en la que declara al quechua como lengua oficial del Perú junto al castellano. En 1989, se agrega el componente intercultural, denominándose Educación Bilingüe Intercultural. Por su parte, las organizaciones de pueblos indígenas, respaldadas por el Convenio 169 de la Organización Internacional del Trabajo, firmado por el Perú en 1994 (OIT, 1989) demandan cada vez más al Estado su derecho a una educación que respete sus lenguas y culturas. Es así, que la actual Constitución Política del Perú (1993) establece que es obligación del Estado fomentar la Educación Bilingüe Intercultural, la que desde el año 2000 se denomina Educación Intercultural Bilingüe. Más recientemente, la Ley General de Educación 28044 (2003) asume la interculturalidad como un principio de la educación nacional, y en el 2016, se aprueba el Reglamento de la Ley 29735, que regula el uso, preservación, desarrollo, recuperación, fomento y difusión de las lenguas originarias del Perú1.

En este contexto el Ministerio de Educación (Minedu, 2016) y en particular, la Dirección General de Educación Básica Alternativa, Intercultural Bilingüe y de Servicios Educativos en el Ámbito Rural, generó el Plan Nacional de Educación Intercultural Bilingüe a $2021^{2}$ que expresa una creciente conciencia de la necesidad de una educación intercultural para todos, no solo para pueblos indígenas, que contribuya a erradicar de la sociedad peruana

\footnotetext{
1 El Peruano, 22 de julio de 2016. Decreto supremo 004-2016-MC que aprueba el Reglamento de la ley 29735.

2 Refrendado por el decreto supremo 006-2016-MINEDU que aprueba la política sectorial de educación intercultural para todas y todos (EIT) y educación intercultural bilingüe para los pueblos originarios (EIB), y la resolución ministerial 629-2016-MINEDU.
} 
prejuicios, estereotipos, discriminación y exclusión, a favor de una convivencia democrática y del desarrollo del país.

Ante estas políticas de educación intercultural bilingüe, el Ministerio de Educación (2016), en el Currículo Nacional, indica procesos de diversificación curricular a nivel regional, local, institucional y de aula desde un enfoque de interculturalidad. También se han elaborado diversos materiales didácticos destinados a diferentes pueblos indígenas en sus lenguas originarias. En relación al presente estudio, cabe señalar que la Región Ucayali, desde 2013, cuenta con un Diseño Curricular Regional de Educación Primaria Intercultural Bilingüe para los pueblos indígenas de su jurisdicción.

\subsection{Planteamiento del problema: dela política educativa a la práctica en aula}

Se asume que una norma, lineamiento o enfoque establecido por el Estado debe ser llevado a la escuela a través de la práctica docente diaria; sin embargo, la experiencia nos muestra que dichas disposiciones se incorporan de modo formal en el discurso o documentos, pero no necesariamente conducen a la práctica deseada. Tapia y Cueto (2017), en una revisión de los estudios sobre desarrollo de currículo y diversos factores asociados al logro de los aprendizajes, concluyen que existe una brecha entre lo que prescribe el currículo oficial y lo que ocurre en las aulas. Recomiendan tomar medidas orientadas a disminuir dicha brecha.

En tal sentido, es importante conocer cómo entienden los docentes los enfoques y contenidos curriculares, y cuánto coinciden o no con sus propias concepciones, pues se sabe que estas influyen sobre su práctica en el aula (Martín, Pozo, Mateos, Martín y Pérez Echeverría, 2014; Trumbull, Scarano y Bonney, 2006). De allí, la relevancia de que en la elaboración de políticas educativas y del currículo se tomen en cuenta las concepciones de los docentes, de tal modo que estas sean más pertinentes y se implementen como producto del diálogo.

A partir de estas consideraciones, nos preguntamos ¿Cómo entienden, las y los docentes, la interculturalidad y cómo se refleja en su práctica de aula y planificación curricular?

Cabe señalar que, ante la complejidad que implica la práctica docente, (García-Cabrero Cabrero, Loredo y Carranza, 2008) en esta investigación asumimos la "práctica en aula» como la manera en que cada docente conduce los procesos de enseñanza y de aprendizaje previstos en la planificación curricular y otros temas emergentes, así como a las interacciones que establece con los estudiantes. De otro lado, la programación curricular es entendida como 
el proceso de planificación curricular a nivel de aula. Implica el planeamiento de las unidades de aprendizaje que orientan el desarrollo de las sesiones de aprendizaje en el aula y que contienen las competencias y capacidades que desarrollaran los estudiantes en un periodo del año escolar.

\subsection{Antecedentes del estudio}

En la revisión de investigaciones previas, en el Perú encontramos tres estudios que exploran las concepciones de docentes y funcionarios de educación sobre interculturalidad y educación intercultural. En el más reciente, León (2014) concluye que, entre los docentes participantes, de nivel inicial, en Puno, Cusco y Ucayali, prevalece un concepto de interculturalidad y educación intercultural centrado en el uso de la lengua materna, con poca atención a aspectos como identidad, cosmovisión y técnicas originarias. Identifica como aspectos positivos la presencia de elementos de la cultura originaria en la ambientación del aula, algunos contenidos, el uso de la lengua en las sesiones de aprendizaje y la participación de sabios de la comunidad en el aula, pero como actividades que no aseguran procesos pedagógicos integrales. El autor advierte falta de claridad en cómo hacer educación intercultural en el nivel pre escolar y dificultad para la programación curricular.

De otro lado, Valdiviezo (2013), en un estudio sobre educación intercultural bilingüe con docentes de tres escuelas del Valle Sagrado de los Incas, Cusco, encuentra variedad y complejidad en las conceptualizaciones de los docentes, algunas que señala como estáticas y otras más fluidas, pero, en general, con una tendencia a hacer eco del discurso oficial de la política intercultural que destaca la comprensión cultural mutua y las relaciones colectivas armoniosas. Desde una perspectiva crítica, advierte que dichas conceptualizaciones invisibilizan las desigualdades, sus causas y consecuencias para diferentes grupos culturales y sociales; por tanto, no cuestionan las relaciones asimétricas de poder. No obstante, encuentra algunas opiniones críticas, especialmente de docentes de origen indígena, y plantea la necesidad de conocer mejor cómo reinterpretan la política de educación intercultural para ponerla en práctica.

Por su parte, Peschiera (2010) encuentra que funcionarios y docentes de diferentes regiones del país expresan discursos sobre interculturalidad de tipo normativo, desde una mirada utópica que no cuestiona las desigualdades ni las relaciones de poder vigentes. La mayoría de dichos actores educativos restringen la EIB a los pueblos indígenas, en la lógica de preservar y rescatar las culturas consideradas de menor prestigio en relación a los contextos hegemónicos. Concluye que, estas concepciones responden a la perspectiva 
funcional de interculturalidad y considera que esta manera de interpretar la interculturalidad sería la principal interferencia para los propósitos de la educación intercultural.

En los tres estudios descritos, prevalece entre los docentes un discurso normativo de la interculturalidad y señalan la ausencia de una perspectiva crítica. También identifican elementos positivos relacionados principalmente con la preservación y afirmación de las lenguas y culturas originarias. Surge la necesidad de realizar más investigaciones para comprender la diversidad en las concepciones de interculturalidad de los docentes y para examinar su correspondencia con la práctica en aula y la planificación curricular, para así realimentar la política del Estado en esta materia, con una base empírica.

\section{MARCO TEÓRICO: CONCEPCIONES E INTERCULTURALIDAD}

En esta sección, se detalla a qué se denomina concepciones en la presente investigación y se presentan diferentes planteamientos sobre qué es interculturalidad con la finalidad de identificar e interpretar el o los que se condicen con la concepción de interculturalidad encontrada en los docentes.

En relación a las concepciones, se enfrenta la dificultad de la utilización de diferentes términos como si fuesen sus sinónimos: creencias, concepciones, interpretaciones, y representaciones para referirse al "pensamiento" que se intenta conocer y comprender, lo que genera cierta confusión (Jiménez y Feliciano, 2006). Creencias y concepciones son los términos más usados como sinónimos. En otros casos, se asume que las concepciones pueden integrar las creencias, los significados, conceptos, proposiciones, preferencias y gustos (Ponte, 1999, citado por Ginocchio, Frisancho y La Rosa, 2015).

Según Giordan y De Vecchi (1995), citados por López-Vargas y BastoTorrado (2010), las concepciones son producto de un proceso personal que integra conocimientos aprendidos en la cultura familiar, en la práctica social, en la escuela, los medios de comunicación, la actividad profesional y social del adulto. Es decir, las concepciones tienen raíces socioculturales y son, a su vez, un factor de socialización que se encuentra en la base de los intercambios psicosociales producidos en el campo de la acción (Rodrigo, Rodríguez y Marrero, 1993); son fruto de procesos dinámicos que integra aspectos socio culturales, cognitivos y emocionales que influyen en la manera de actuar de las personas (Buendía, Carmona, González y López, 1999).

Por su parte, Arbeláez (2010) plantea que las concepciones: 
que constituye un marco de significación. En las concepciones expresadas, podemos reconocer muchas acciones, muchos momentos significativos y procesos de construcción mental impactados por vivencias individuales y colectivas que afectan su significado. Toda concepción tiene dos componentes: uno tiene que ver con el qué se concibe y el otro con cómo se concibe. Conforman los marcos de referencia desde los cuales actúa el ser humano, son el prisma a través del cual percibe y el contexto sobre el cual procesa la información (p. 4).

Sobre la base de lo expuesto, en la presente investigación, las «concepciones» refieren a la manera en cómo, las y los docentes, de manera personal, entienden la interculturalidad, en qué consiste y cómo se manifiesta.

Respecto a la interculturalidad, autores como López (2001) y Walsh (2009) coinciden en que este término surgió como respuesta a la función homogeneizadora de los Estados nacionales de América Latina mediante la educación oficial, empeñados en forjar una identidad nacional en países caracterizados por su diversidad étnica, cultural y lingüística, en desmedro de los pueblos indígenas, al desvalorizar sus culturas originarias.

A la interculturalidad se le atribuye diferentes significados, dependiendo del actor social que la plantea, como organizaciones indígenas, académicos o autoridades educativas. La interculturalidad llevada a la práctica educativa es motivo de discusión en la agenda de los movimientos indígenas, desde los años 80 , con respuestas diferentes según su experiencia histórica y expectativas. En el Perú, los pueblos amazónicos asumen la Educación Intercultural Bilingüe (EIB) como una reivindicación de sus derechos étnicos, culturales y lingüísticos, mientras que algunos pueblos andinos la miran con cierto temor y desconfianza (Zavala, Cuenca y Córdova, 2005), sobre todo, porque la EIB continúa siendo una educación para pueblos indígenas.

Encontramos que, en general, el concepto de interculturalidad alude a la relación e intercambio de costumbres, valores y conocimientos, entre personas y pueblos de culturas diferentes. Sin embargo, un análisis de las condiciones en que las personas y pueblos de diferentes culturas se relacionan da cuenta de una interculturalidad funcional y otra crítica, dependiendo de factores históricos, sociales, económicos y políticos del contexto (Tubino, 2005; Walsh, 2000).

La interculturalidad funcional preconiza relaciones armoniosas respetando las diferencias, pero sin cuestionar las situaciones de inequidad ni sus causas estructurales que perjudican a minorías étnicas y pueblos originarios o indígenas, considerados de menor desarrollo y valor que la cultura hegemónica. En cambio, la perspectiva crítica de interculturalidad considera fundamental conocer, cuestionar y modificar las relaciones de poder que generan condiciones y relaciones inequitativas. Plantea generar condiciones de igualdad que 
garanticen un diálogo y soluciones respetuosas a los conflictos y tensiones propias de la convivencia humana (Fornet-Betancourt, 2000).

En igual sentido, Walsh (2000) afirma que:

[...] en los encuentros entre personas, elementos o prácticas culturales, las inequidades sociales, étnicas, económicas y políticas de la sociedad no desaparecen. Sin embargo, es en este espacio fronterizo de relación y negociación que también se construyen y emergen nuevas estrategias, expresiones, iniciativas, sentidos y prácticas [inter]culturales, que desafían la homogeneidad, el control cultural y la hegemonía de la cultura dominante (p. 12).

Por su parte, Zúńiga afirma que la interculturalidad:

[...] posee carácter desiderativo; rige el proceso y es a la vez un proceso social no acabado sino más bien permanente, en el cual debe haber una deliberada intención de relación dialógica, democrática entre los miembros de las culturas involucradas en él y no únicamente la coexistencia o contacto inconsciente entre ellos. Esta sería la condición para que el proceso sea calificado de intercultural (Zúñiga, 1995, citada por Zúńiga y Ansión, 1997).

Es claro que, sin cuestionar las raíces estructurales de las inequidades entre personas y pueblos, la interculturalidad se convierte en un discurso y una práctica que puede producir cambios superficiales o insuficientes que no logren alterar un sistema social, económico y político injusto. Asumir una perspectiva de interculturalidad crítica exige cambios profundos en las estructuras de poder, como condición para una convivencia respetuosa, participativa y en diálogo permanente, como un proyecto nacional integral y no solo de la educación ni solo para pueblos originarios. Por lo tanto, la práctica de una interculturalidad crítica involucra diferentes dimensiones: social, cultural, lingüística, ética, política y económica.

También es importante identificar dos tipos de discurso sobre interculturalidad: un discurso descriptivo y otro normativo. Por un lado, el discurso descriptivo de interculturalidad refiere hechos empíricamente verificables respecto a la convivencia de personas o pueblos con diferentes prácticas culturales en un espacio físico, enfrentando tensiones o conflictos. Por otro lado, el discurso normativo del «deber ser» de la interculturalidad se produce en la filosofía y la educación. La filosofía plantea la interculturalidad como un proyecto ético-político de democracia inclusiva de la diversidad. En la educación, el discurso normativo de la interculturalidad se expresa como principio, y enfoque curricular (Tubino y Zariquiey, 2004).

Tubino (2004), en «Interculturalidad para todos: ¿̨un slogan más?», plantea que: 
Desde estos discursos sobre la interculturalidad existentes en el Perú actual creemos que es posible y necesario construir un concepto que - recogiendo los principales aportes de cada uno de ellos - nos sirva para identificar los lineamientos claves que hay que seguir para ofrecer una educación intercultural abierta a las diferencias y flexible a las circunstancias a nivel de todo el sistema educativo nacional (p. 3).

En igual sentido, Peschiera (2010) cita a Zavala, Cuenca y Córdova (2005) con quienes coincide en que los discursos, normativo y descriptivo, no son excluyentes ni contradictorios entre sí y plantea que «es necesario identificar los lineamientos claves para consensuar a la interculturalidad, de forma abierta, crítica y flexible» (p. 17).

\section{Metodología}

Este estudio utiliza un enfoque metodológico cualitativo para profundizar en las concepciones (Simons, 2009) que los docentes en escuelas de Lima y Ucayali han elaborado sobre interculturalidad y cómo dichas concepciones orientan su práctica en aula para implementar una educación intercultural.

El objetivo general del estudio fue identificar las concepciones de interculturalidad que los docentes han elaborado y cómo están presentes en la programación curricular y en su práctica de aula en escuelas interculturales bilingües shipibo-castellano en Lima y en Ucayali. Para ellos, se plantearon tres objetivos específicos: (1) reconocer en el discurso de los docentes comprendidos en el estudio la concepción de interculturalidad que orienta su labor pedagógica; (2) identificar cómo sus concepciones de interculturalidad se reflejan en su práctica en aula; e (3) identificar cómo sus concepciones de interculturalidad están presentes en la programación curricular de corto plazo.

\subsection{Participantes}

Participaron 15 docentes de cuatro escuelas públicas que atienden a niños y niñas de comunidades shipibas: dos en Lima (capital del país), donde reside un grupo de familias shipibas migrantes, y otras dos en Ucayali, que albergan a familias shipibas en su región de origen (ver Tabla 1). Los docentes recibieron información sobre los objetivos y procedimientos de la investigación, así como sobre la forma de su participación, firmando un consentimiento informado. 
Nora Cépeda García, Dina Castro Burgos, Pilar Lamas Basurto

Tabla 1. Características demográficas de los docentes participantes $(\mathrm{N}=15)$

\begin{tabular}{lccc}
\hline Características demográficas & \multicolumn{2}{c}{ Institución educativa } & Total \\
& Ucayali & Lima & \\
\hline Docentes & 5 & 10 & 15 \\
Escuela inicial & 2 & 4 & 6 \\
Escuela primaria & 3 & 6 & 9 \\
Lugar de nacimiento & & & \\
$\quad$ Ucayali & 5 & 3 & 8 \\
Lima & - & 5 & 5 \\
Cusco & - & 1 & 1 \\
Jauja & - & 1 & 1
\end{tabular}

Sexo

Femenino

$3 \quad 9 \quad 12$

Masculino

Idioma

Castellano

$\begin{array}{lll}- & 6 & 6 \\ - & 1 & 1 \\ 5 & 3 & 8\end{array}$

Shipibo/Castellano

Grupo étnico

Shipibo

5

8

Mestizo

Habilidad para hablar shipibo

Muy bien

$\begin{array}{lll}3 & 3 & 6 \\ 2 & - & 2 \\ - & 2 & 2 \\ - & 5 & 5\end{array}$

Nivel y área de especialidad

Educación inicial

Educación primaria

$\begin{array}{lll}2 & 4 & 6 \\ 3 & 6 & 9\end{array}$

Educación secundaria EIB

3 9

* Algunos docentes tienen más de un área de especialidad.

Fuente: Datos obtenidos de las entrevistas. 


\subsection{Procedimientos e instrumentos}

Para lograr los objetivos planteados, utilizamos tres procedimientos con sus respectivos instrumentos: entrevista semiestructurada, observación no participante en aula y análisis documental de unidades de aprendizaje. Los resultados de estas tres fuentes de datos fueron analizados independientemente y triangulados para arribar a las conclusiones generales del estudio.

Se inició con las entrevistas sobre la concepción de interculturalidad de cada docente y datos demográficos. En la codificación inicial de las entrevistas, se hallaron 63 categorías, que fueron luego organizadas por temas afines y se redujo el número de categorías según los criterios de que cada una sea exhaustiva, excluyente, significativa y replicable (Ruiz, 1996). Este proceso concluyó en la identificación de categorías comunes en las concepciones de las y los docentes de Lima y Ucayali; y de categorías únicas consideradas solo por docentes de uno de los lugares. Dichas categorías orientaron el posterior análisis de las observaciones en aula y el análisis de las unidades de aprendizaje (Hesse-Biber y Leavy, 2011).

Con los resultados preliminares, se realizó un grupo focal, en cada región, para socializar los hallazgos con los docentes participantes, confirmando, aclarando y complementando el análisis realizado.

Usando las categorías identificadas en las entrevistas, se procedió con la observación de las aulas de las y los docentes para identificar cómo su concepción sobre interculturalidad se refleja en su práctica. Para ello, se recogió información sobre uso de lenguas, tratamiento de contenidos, procedimientos, prácticas propias de la cultura shipiba y aportes de otras culturas, la ambientación del aula, el material educativo, las interacciones entre docente y estudiantes, y entre estudiantes. Luego de ello, se procesó la información de las observaciones y se analizó su relación con la concepción de interculturalidad que cada docente expresó en la entrevista.

Para identificar cómo cada docente incluye su concepción de interculturalidad en la programación curricular de aula, utilizamos el análisis documental de unidades de aprendizaje. En cada unidad, se analizaron los componentes curriculares: competencias, capacidades e indicadores de las diferentes áreas, incluyendo, siempre que fue posible, las actividades de aprendizaje a realizar durante el periodo previsto en la unidad. Cabe precisar que, para la planificación, los docentes en las escuelas de Lima utilizan el Programa Curricular Nacional de Educación Primaria (Ministerio de Educación del Perú, 2016) el mismo que presenta los aprendizajes a lograr en las áreas de: Comunicación, Matemática, Personal Social, Ciencia Tecnología y Ambiente, Educación 
Física, Educación Religiosa, Arte y Cultura, inglés y Castellano como segunda lengua. En las escuelas de Ucayali, por su parte, tienen como referente el Diseńo Curricular de Educación Primaria Intercultural Bilingüe para los pueblos indígenas de la Región Ucayali (2013) que incorpora dos nuevas áreas: Territorio e Identidad y Espiritualidad, que responden a las características sociales y culturales de su contexto además del área de «Lengua materna en lengua indígena».

\section{Resultados}

En esta sección, presentamos las categorías conceptuales identificadas en las concepciones de interculturalidad de los docentes, distinguiendo las que son comunes de las que son únicas en cada grupo de docentes: Lima y Ucayali. Asimismo, presentamos las prácticas de aula y aspectos de la programación curricular que se condicen con cada categoría conceptual.

De las entrevistas, emergieron tres categorías conceptuales comunes que tienen los docentes de las escuelas de Lima y Ucayali, como parte de su concepción de interculturalidad. Para ellos, la interculturalidad implica (a) afirmar y valorar lo propio, (b) el encuentro con otras lenguas y culturas, y (c) el intercambio entre culturas. La primacía de estas categorías se ratificó en los grupos focales posteriores a las entrevistas.

Es así que, para los docentes de las escuelas participantes, la interculturalidad empieza por «afirmar y valorar lo propio», una categoría conceptual que agrupa respuestas que destacan la importancia de valorar primero su cultura para relacionarse con otros con respeto y construir la interculturalidad: «yo pienso que primero lo nuestro, conocer nuestra propia cultura y costumbres y luego la de otros» (docente shipibo, Ucayali). En los grupos focales, los docentes ratificaron que afirmar y valorar la cultura propia es la base y condición fundamental para el intercambio con otros.

La segunda categoría «encuentro con otras lenguas y culturas», integra ideas relacionadas con reconocer diferencias con otras etnias o grupos, como la lengua, costumbres y cosmovisiones: «Para mí es hablar las dos lenguas. Hablar mi propia lengua y aprender la de otro a partir de la práctica respetuosa del otro" (docente shipiba, Lima). En los grupos focales, los docentes ratificaron este significado y enfatizaron la importancia de la afirmación de la lengua shipiba y de aprender otras lenguas además de la propia.

La tercera categoría, «intercambio entre culturas», se refiere al intercambio respetuoso de conocimientos, compartir y dialogar con diferentes grupos étnicos y lingüísticos: "Compartir conocimientos con otras personas que no son de mi grupo. Puede ser personas mestizas, japonés, chinos; yo comparto 
con ellos mi cosmovisión, y compartimos la forma en que cada uno ve el mundo» (docente shipibo, Ucayali). En los grupos focales, destacaron a la interculturalidad como una oportunidad para intercambiar conocimientos, creencias y costumbres entre miembros de diferentes culturas, aprender de otros e incorporar nuevos elementos a su experiencia: «Ser intercultural, creo, no solamente es conocer, sino mirar más allá, cómo podemos mejorar. En este mundo globalizado ya no solo mirar a los pueblos originarios sino ver... otros países» (docente mestiza, Lima).

Es importante señalar que en ambos grupos focales los docentes diferenciaron encuentro de intercambio, reconociendo que si bien un encuentro entre culturas y entre lenguas, puede ser beneficioso porque permite conocer sobre otros grupos, no involucra necesariamente, un auténtico intercambio, es decir aprender uno de otro.

Como categorías únicas, docentes de Ucayali mencionaron que el encuentro entre diferentes culturas genera cambios en las costumbres y modos de ver las cosas. Por otro lado, los docentes de Lima, destacaron el respeto y la no discriminación como comportamientos interculturales: «... yo creo que es el respeto a las culturas, el de un niño mestizo, de un nińo shipibo, porque aquí no se discrimina a ningún niño» (docente mestiza, Lima).

Podemos afirmar que las concepciones de interculturalidad de los docentes de Lima y de Ucayali, shipibos y no shipibos, enfatizan de manera más significativa la afirmación y valoración de la propia cultura como condición fundamental para relacionarse con otras culturas, con muy alto compromiso con la conservación de la lengua shipiba. No obstante, la mayoría de docentes incluye elementos de las categorías «encuentro con otras culturas» e «intercambio entre culturas", mostrando en sus concepciones diferentes niveles de complejidad.

De acuerdo a la metodología, se observó en aula a los docentes entrevistados para identificar como su concepción sobre interculturalidad se refleja en su práctica. Es posible decir que existe relación entre lo que cada docente expresó y lo que hace en el aula. A continuación, presentamos ejemplos que evidencian esta relación y para ello exponemos lo que respondieron algunos maestros sobre interculturalidad y lo que se halló en sus respectivas aulas.

En la siguiente afirmación sobre interculturalidad se pueden identificar elementos de las tres categorías mencionadas:

Para mí, es hablar las dos lenguas. Hablar mi propia lengua y aprender la de otro a partir de la práctica respetuosa del otro. La práctica de todo lo que es relacionado a otras culturas. Para nosotros la interculturalidad es compartir con respeto, sin práctica no se puede hablar sobre interculturalidad (docente shipiba, Lima). 
En esta aula de nivel inicial, la mayoría de estudiantes pertenecen a la comunidad shipiba, pero nacieron en Lima, muy pocos son mestizos, todos hablan castellano. Se observó que la docente dirige las actividades en castellano, pero aprovecha todas las oportunidades (saludo, despedida, días de la semana, canciones) para que niñas y niños aprendan el shipibo como segunda lengua (L2) incluyendo a niños mestizos. De esta manera afirma el valor de la lengua shipiba. Por otra parte, afirma la identidad étnica de los shipibos mostrando acogida a los mestizos:

Maestra: Canten fuerte, por qué no cantan fuerte (en shipibo), todos nos sentimos orgullosos de nuestra comunidad shipiba que queremos tanto. ¿Quiénes son de la comunidad shipiba? [Niñas y niños, levantan la mano, incluso un niño mestizo]

Maestra: [se acerca al niño mestizo] Tú no eres. [de inmediato se dirige a todos]: Pero él nos quiere como si lo fuera y nosotros lo queremos mucho.

Una docente de Lima, mestiza de origen andino, se refirió a la interculturalidad como: «Es una mezcla hermosa de costa, sierra y selva. Es rico conocer lo que saben los niños, mezcla de diferentes culturas. Tengo niños de Puno y de Huancayo, no hablan quechua, pero si tienen la cultura, por ejemplo, el huayno, en su casa tienen otra cultura. He observado que no se adaptan a los shipibos».

Observamos que esta docente de primer grado apunta cada día las nuevas palabras en shipibo que aprende con los niños y colegas; participa en la clase de shipibo (L2) a cargo de una docente shipiba y entona canciones en shipibo. A su vez, enseñó a niños y niñas, mestizos y shipibos a cantar un huayno en castellano y danzas andinas.

La siguiente concepción de interculturalidad menciona el compartir y el entendimiento como formas concretas del intercambio, para lo cual es condición la afirmación de lo propio: "Compartir conocimientos con otras personas que no son de mi grupo.... Mi cosmovisión y la forma en que cada uno ve el mundo.... Pero yo pienso que primero lo nuestro, conocer nuestra propia cultura y costumbres y luego la de otros. Yo pienso que la interculturalidad nos permite llegar a entendernos con los hermanos mestizos» (docente shipibo, Ucayali).

En esta aula de quinto y sexto grados, participan algunos estudiantes mestizos. Se observó que el docente aprovecha los acontecimientos de la comunidad para que sus estudiantes, shipibos y mestizos, comprendan, afirmen y valoren la cultura shipiba. Por ejemplo, un niño falleció y todos participaron 
de las ceremonias funerarias shipibas. Al día siguiente, este docente inició la clase explicando en lengua shipiba que cuando alguien muere en la comunidad shipiba «muere un hermano». En otra oportunidad, trabajó el uso de letra mayúscula utilizando los nombres shipibos de niños y niñas, les explicó: «todos nuestros nombres vienen de los animales». También se observó en esta aula, actitud de acogida a personas de diferentes culturas: dos practicantes de educación de la etnia kunicuy (Purús), una joven suiza y otra alemana, ambas voluntarias que enseñan inglés en la escuela, ninguna de las cuales habla shipibo, por lo que la comunicación es en castellano. Con esta experiencia, el profesor anima a sus estudiantes: «Queremos ser trilingües. Cuando hablo en shipibo, cuando hablo con el presidente en castellano, cuando me voy a EE.UU. saludo al presidente Obama en inglés. Queremos ser así, del mundo».

Durante las observaciones, se constató que los docentes que expresaron de manera más consistente su concepción sobre interculturalidad muestran un desempeño más coherente con lo que piensan. Mientras que, quienes expresaron ideas un tanto difusas tienen dificultad para concretarlas en su práctica. Llama la atención que la mayoría de acciones o comportamientos más coherentes con lo que dicen sobre interculturalidad no son programadas en las unidades de aprendizaje, especialmente en las escuelas de Lima, más bien emergen como acciones informales, sea dentro o fuera de una sesión de clase.

Por otro lado, y siguiendo la metodología, en el análisis de las unidades de aprendizaje de Educación Inicial y Primaria, se aprecia que, en las escuelas de Lima, las competencias y capacidades que se pretenden desarrollar en los y las estudiantes son las que prescribe el currículo nacional; es decir, son aprendizajes que todos los niños peruanos sin distinción de su origen étnico o cultura deberían alcanzar. Sin embargo, es claro el propósito de esta escuela en preservar la lengua shipiba en todos los grados de inicial y primaria, ya que en la programación del área curricular denominada Segunda Lengua (L2) se enseña la lengua shipiba. Esto coincide con las concepciones de interculturalidad, expresadas por los docentes, que buscan la afirmación cultural a través del aprendizaje o preservación de la lengua originaria, más aún considerando que en estas escuelas los estudiantes de los primeros grados no tienen el shipibo como lengua materna, pues ya nacieron en Lima.

Cabe señalar también que en las escuelas de Lima se encontró solo dos enunciados referidos a la capacidad de desarrollar la valoración positiva de la cultura shipiba como punto de partida para la relación intercultural expresada por los docentes. Se lee en la unidad de aprendizaje analizada la siguiente capacidad: Se relaciona interculturalmente con otros desde su identidad y enriqueciéndose mutuamente (docente mestiza, $3^{\circ}$ grado, Lima). Asimismo, 
en otra unidad, el indicador de aprendizaje señala: Expresa agrado y orgullo por la vivencia y manifestación educativa y su comunidad y por sentirse parte de estos grupos (docente shipiba, $2^{\circ}$ grado, Lima).

La planificación de las unidades de aprendizaje de Educación Inicial y Primaria en las escuelas de Ucayali, se rige por el Currículo Intercultural Bilingüe para los Pueblos Indígenas de la Región Ucayali (CIBPIRU, 2013), que incorpora dos nuevas áreas al currículo nacional: Territorio e Identidad y Espiritualidad. En el área Territorio e Identidad, destacan capacidades relativas al conocimiento de la historia local (pueblo/comunidad), las características organizativas y mecanismos de la toma de decisiones propios de las comunidades. Asimismo, se aprecia el reconocimiento de las actividades económicas originarias (ej. caza, pesca, recolección, elaboración de recipientes y tejidos). En el área Espiritualidad, destaca la capacidad relativa al establecimiento de una relación del hombre shipibo y la naturaleza basada en la reciprocidad, el respeto, la búsqueda de equilibrio en la convivencia con la naturaleza y la concepción mágica sobre ella.

Es así que, en las escuelas de Ucayali, la concepción de interculturalidad de los docentes que está presente en todas las unidades de aprendizaje es la de la afirmación de la cultura propia, vinculada a las capacidades de preservación y valoración de su lengua, cosmovisión, espiritualidad y conocimientos. Es necesario resaltar que, en el currículo regional, como en las unidades de aprendizaje, se hace evidente la valoración de los conocimientos ancestrales de la comunidad shipiba sin dejar de lado el conocimiento que proviene de la cultura occidental (hegemónica) a través de los contenidos de ciencias del currículo nacional. Se observa:

\section{Area curricular Ciencia y Ambiente}

Capacidad: identifica los animales en el ecosistema e investiga relatos de su pueblo que explican el origen y los clasifica según el hábitat.

Capacidad: Comprende la importante función que cumplen los animales en el ecosistema e identifica las especies en peligro de extinción. (Ucayali, 3er y 4to grado de Primaria: unidad didáctica junio)

En resumen, en las unidades de aprendizaje analizadas, tanto de Lima como de Ucayali destaca el propósito de la afirmación de la cultura shipiba a través de la consideración de competencias y capacidades orientadas al aprendizaje o preservación de su lengua y Ucayali rescata conocimientos ancestrales y propios de la cultura shipiba incluyendo capacidades específicas en las áreas contempladas en su currículo regional. 


\section{Discusión}

De acuerdo a los objetivos y hallazgos de la investigación, en esta sección, planteamos algunas reflexiones a partir de las concepciones de interculturalidad construidas por las y los docentes participantes. Nos referiremos a cuatro temáticas. Las dos primeras emergen consistentemente de las tres fuentes de datos recogidos, entrevistas, observaciones en aula y unidades de aprendizaje: (1) la afirmación de la cultura propia [shipiba] como condición para la interculturalidad; (2) la interculturalidad como un intercambio entre culturas. Las otras dos temáticas surgen de analizar dichas concepciones en relación a las definiciones teóricas sobre interculturalidad presentadas en el marco conceptual del estudio: (3) el predominio de una perspectiva funcional y normativa de la interculturalidad; y (4) la interculturalidad como un proceso dinámico.

\subsection{Sobre la afirmación de la cultura propia}

Encontramos que los docentes participantes, tanto en su discurso como en su práctica, con distinta intensidad, concuerdan en que la afirmación cultural es una condición fundamental para establecer un diálogo con otras culturas. En este proceso, la lengua shipiba y su aprendizaje merece especial relevancia. Este hallazgo concuerda con los estudios de León (2014) y Valdiviezo (2013) quienes encontraron que los docentes tienen una alta valoración de las lenguas originarias (shipibo, quechua) como elemento indispensable para el mantenimiento o revitalización cultural, lo cual es muy positivo.

En las cuatro escuelas, los docentes incluyen elementos de la cultura y lengua shipiba en la ambientación, en las actividades de aula y en las unidades de aprendizaje, orientadas a valorar la cultura originaria, prácticas que son más consistentes cuando la escuela forma parte de un entorno con predominio cultural shipibo. En Ucayali, la afirmación cultural en la programación de unidades de aprendizaje tiene dos mediadores importantes definidos por el Currículo Regional, el desarrollo de las competencias comunicativas en su lengua nativa y el aprendizaje y el rescate de la historia, conocimientos y expresiones culturales shipibas en las áreas curriculares, especialmente en la de Espiritualidad, Territorio e Identidad. Asimismo, los docentes utilizan la lengua shipiba para comunicarse con los estudiantes y padres de familia en interacciones fuera del aula (ej. durante los recesos, celebraciones, y reuniones).

En contraste, la afirmación cultural en las escuelas de Lima presenta enormes retos por encontrarse en un contexto con una cultura mestiza hegemónica y que tiene como referente el Currículo Nacional. En tal situación, la enseñanza del shipibo como segunda lengua se torna en un elemento crucial 
de la preservación de la cultura debido a que las nuevas generaciones (hijos de migrantes shipibos) que asisten a la escuela no tienen el shipibo como lengua materna. No obstante, los esfuerzos resultan limitados debido a que, según indicaron los docentes shipibos y los mestizos, no cuentan con las herramientas pedagógicas para enseñar a leer y escribir en lengua shipiba e incorporar elementos de la cultura shipiba en las actividades curriculares. En cierta forma, las escuelas de la comunidad shipiba en Lima podrían resultar espacios de preservación cultural «aislado», tanto de su pueblo originario como del contexto de la ciudad, a pesar de los esfuerzos de las familias migrantes shipibas por incorporarse a la vida en Lima contribuyendo a la actividad económica y social. Esta situación evidencia la necesidad de implementar las políticas de educación intercultural para todos, ya que en Lima encontramos poblaciones migrantes con diversas lenguas y prácticas culturales, hacia quienes persisten actitudes y comportamientos discriminatorios y racistas.

Al plantear la afirmación de la cultura propia como una condición previa y fundamental para que se dé la interculturalidad (entendida como el intercambio respetuoso entre culturas), los docentes participantes en este estudio reconocen la existencia de relaciones sociales inequitativas entre el pueblo shipibo y «la cultural nacional», que es como ellos llaman a la cultura mestiza hegemónica. Más aún, asumen la Educación Intercultural Bilingüe como una reivindicación de sus derechos étnicos, culturales y lingüísticos (Zavala, Cuenca, y Córdova, 2005). Sin embargo, es necesario mencionar que ninguno de los docentes hizo mención a las razones de estas relaciones de inequidad.

Cabe apuntar que el Currículo Nacional de Educación Básica Regular vigente prescribe que, al término de la educación básica, «el estudiante valore, desde su individualidad e interacción con su entorno sociocultural y ambiental, sus propias características generacionales, las distintas identidades que lo definen, y las raíces históricas y culturales que le dan sentido de pertenencia» (Minedu 2017, p. 14). En este sentido, se podría decir que las prácticas de aula realizadas con el propósito de afirmar la cultura shipiba, observadas en todas las escuelas participantes, contribuyen al logro de este objetivo.

Los hallazgos de este estudio afirman la necesidad de tomar en cuenta las experiencias que aportan los docentes desde su entendimiento de la interculturalidad elaborado a partir de su práctica cotidiana, en el aula y en su comunidad, en lugar de partir de un concepto normativo o ideal de la interculturalidad, más bien se tendría que partir de la interculturalidad de hecho o de facto para, desde ahí, apuntar a un cambio social para el futuro (Zavala, Cuenca y Córdova, 2005). 


\subsection{Sobre la interculturalidad como un intercambio entre culturas}

Los docentes shipibos y mestizos, al ser entrevistados, en Ucayali y en Lima, se refirieron a la interculturalidad como el intercambio entre culturas, entendido como el compartir de conocimientos entre diferentes grupos étnicos y culturales (nacionales y de otros países). El respeto, la comunicación, la disposición a aprender de otros y el diálogo entre culturas forman parte de sus concepciones de interculturalidad. Estas concepciones coinciden con planteamientos que sostienen que la interculturalidad es la relación, comunicación y aprendizaje entre personas, grupos, conocimientos, valores y tradiciones distintas, orientada a generar, construir y propiciar un respeto mutuo (Walsh, 2000); y que la interculturalidad no es un concepto, sino una manera de comportarse (Tubino, 2005). Concordamos en que es un hecho complejo e irreductible a categoría teórica, ya que apunta a una forma de ser en el mundo (Tubino, 2015). Las concepciones de los docentes coinciden, así mismo, con el enunciado del enfoque transversal de interculturalidad que define el Currículo Nacional de la Educación Básica como un proceso dinámico y permanente de interacción e intercambio entre personas de diferentes culturas (Minedu, 2017). Sin embargo, esta idea de interculturalidad descrita por las y los docentes de las escuelas de Lima y Ucayali no se reflejan consistentemente en sus prácticas de aula ni en las unidades de aprendizaje.

En relación a la programación curricular de aula, podría pensarse que la disposición para el diálogo intercultural y el respeto a otras culturas en tanto son aprendizajes de carácter actitudinal no son susceptibles de planificación en la unidad de aprendizaje y quedan libradas al ámbito de la formación a través de las interacciones entre docentes y estudiantes en el día a día por modelamiento. Ello coincide con lo observado, en que las actividades más próximas a prácticas interculturales se dan en espacios no formales, dentro o fuera del aula. Con todo, no deja de ser una limitación para la implementación de una educación intercultural, pues al no planificar intencionalmente sesiones de aprendizaje para el desarrollo de la capacidad de intercambio o diálogo intercultural se pierden oportunidades para la sensibilización, reflexión y discusión con los estudiantes, e incluso entre docentes.

\subsection{Sobre la visión normativa y funcional de la interculturalidad}

En las concepciones de los docentes participantes, encontramos el predominio de una perspectiva normativa de la interculturalidad, discurso del «deber ser», basado en una utopía por construir. Tubino (2004) alerta que, si bien este discurso orienta hacia dónde dirigir los esfuerzos, también pone en riesgo la 
capacidad de percibir y cuestionar las situaciones y condiciones de desigualdad que interfieren e impiden avanzar hacia actitudes y relaciones interculturales deseables. Este predominio podría explicar que, tanto en la práctica observada como en las unidades de aprendizaje analizadas, no incluyeran la existencia de desigualdades o conflictos sociales de origen étnico cultural en forma explícita. Al invisibilizar el conflicto, el logro de la meta de la interculturalidad se hace más difícil ya que los factores que la impiden (marginalización, racismo) no son abordados críticamente (Peschiera, 2010).

Por otra parte, como en los estudios de Valdiviezo y Valdiviezo (2008) y Peschiera (2010), la mayoría de concepciones sobre interculturalidad que manifiestan los docentes son más próximas a la perspectiva funcional que, según la definen Tubino (2004) y Walsh (2009), propugna el diálogo, la convivencia y la tolerancia sin cuestionar las causas estructurales de las inequidades sociales, políticas y económicas entre culturas, como lo plantea la perspectiva crítica de interculturalidad.

\subsection{La interculturalidad como un proceso dinámico}

De las concepciones sobre interculturalidad manifestadas por los docentes, emerge la imagen de un "continuum en espiral» en el desarrollo de la interculturalidad como un proceso que involucra la afirmación de la cultura propia, el encuentro entre culturas y el intercambio, respetando la identidad de las culturas de origen. En ese sentido, es un proceso a través del cual se afirma lo propio y el respeto por otros, la necesidad de reconocimiento mutuo en un contexto de equidad, la disposición para aprender de otros y, a la vez, aportar a que otros aprendan, no como un estado estático que se alcanza de manera definitiva, sino como una nueva manera de percibirnos y de convivir.

Considerar de esta manera a la interculturalidad puede ser de mucha utilidad para trabajar con las y los docentes la implementación de una educación intercultural para todos, que se sustente en el enfoque crítico de la interculturalidad (Walsh, 2009). De esta manera, constituiría un proceso que implique una dinámica permanente de búsqueda y encuentro, y la construcción de un espacio de convivencia intercultural donde la pluralidad es la norma y donde los elementos negativos que atentan contra esa convivencia son identificados y tratados a nivel individual y colectivo (Leiva, 2010).

Se trata de que la valoración positiva hacia la interculturalidad se potencie, pase de considerarse un buen deseo dentro de las escuelas a convertirse en un proyecto social, cultural, ético y político nacional al cual todos podemos aportar. 


\section{CONCLuSiones}

Consideramos que este estudio cumplió el propósito de recoger las concepciones de los docentes sobre interculturalidad y describir el modo como se refleja en su práctica educativa cotidiana y la programación curricular de aula. A continuación, presentamos nuestras conclusiones:

- Tanto en Lima como en Ucayali, las y los docentes reconocen que una condición fundamental para el intercambio entre culturas es la afirmación de la cultura propia, sobre la cual se construyen relaciones interculturales. En las observaciones y en el análisis de las unidades de aprendizaje, se encontraron elementos de afirmación cultural y alta valoración de la cultura shipiba, especialmente la preservación de la lengua. Esta afirmación cultural se da en diferentes formas e intensidades en cada escuela, que puede explicarse por los ámbitos geográficos y socio culturales diferentes, así como por la presencia de docentes shipibos y no shipibos, en cada caso.

- Así como las y los docentes expresan convicción sobre la necesidad de la afirmación de la cultura propia, también entienden que la interculturalidad abarca el intercambio entre pueblos amazónicos y andinos, con la cultura nacional y con otras culturas del mundo. Señalan, así mismo, la importancia de aprender otras lenguas para facilitar la comunicación.

- Se identificó que las y los docentes tienen un entendimiento y una actitud positiva hacia la interculturalidad que tratan de incluir en su práctica en aula; sin embargo, aún no llegan a reflejar consistentemente los alcances expresados en sus concepciones. Manifestaron que necesitan ampliar su conocimiento y contar con herramientas pedagógicas para enseńar la primera y la segunda lengua, cómo hacer dialogar las diferentes culturas en cada área curricular y cómo trabajar la interculturalidad transversalmente (Escarbajal y Leiva, 2017).

- Si bien, en las concepciones de las y los docentes participantes en la investigación, se reconoce el predominio de una perspectiva normativa de la interculturalidad orientada a describir cómo debe ser la interculturalidad, otorgándole un carácter utópico, se puede reconocer las relaciones de inequidad entre grupos étnicos y sus culturas, lo que impiden las relaciones interculturales. Esto indica la presencia de algunos elementos críticos en las concepciones de los docentes. Por ello, consideramos que las políticas educativas, elaboradas en diálogo con las concepciones de los docentes, podrían contribuir a que docentes y estudiantes profundicen y consoliden concepciones y prácticas desde una perspectiva de interculturalidad crítica. 


\section{REFERENCIAS BIBLIOGRÁFICAS}

Arbeláez, R. (2010). En el reconocimiento de las concepciones docentes se encuentra el camino del mejoramiento continuo de la calidad docente. Revista Docencia Universitaria, 2(2). Recuperado de http://revistas.uis.edu. co/index.php/revistadocencia/article/view/1378/1790

Buendía, L., Carmona, M., González, D. y López, R. (1999). Concepciones de los profesores de Educación Secundaria sobre evaluación. Educación XX1, 2, 125-154. https://doi.org/10.5944/educxx1.2.0.377

Congreso Constituyente Democrático (1993). Constitución Política del Perú. Lima. Recuperado de www4.congreso.gob.pe/comisiones/1996/constitucion/cons1993.htm

Congreso de la República del Perú (2003). Ley General de Educación № 28044. Lima. Recuperado de http://www.minedu.gob.pe/p/ley_general_de_educacion_28044.pdf

Dirección Regional de Educación de Ucayali (2013). Diseño Curricular de Educación Primaria Intercultural Bilingüe para los pueblos indigenas de la Región Ucayali. Lima: Minedu.

Escarbajal, A. y Leiva, J. J. (2017). La necesidad de formar en competencias interculturales como fundamento pedagógico: Un estudio en la región de Murcia (España). Profesorado. Revista de Curriculum y Formación de Profesorado, 21(1), 281-293. Recuperado de http://www.redalyc.org/ $\mathrm{pdf} / 567 / 56750681013 . \mathrm{pdf}$

Fornet-Betancourt, R. (2000). La interculturalidad a prueba. Recuperado de http://www.uca.edu.sv/filosofia/admin/files/1210106845.pdf

García-Cabrero, B., Loredo, J. y Carranza, G. (2008). Análisis de la práctica educativa de los docentes: pensamiento, interacción y reflexión. Revista Electrónica de Investigación Educativa, Especial. Recuperado de http:// redie.uabc.mx/NumEsp1/contenido-garcialoredocarranza.html

Ginocchio, L., Frisancho, S. y La Rosa, M.I. (2015). Concepciones y creencias docentes sobre la democracia en el colegio. Revista Peruana de Investigación Educativa, (7), 5-29. Recuperado de https://es.calameo.com/ $\mathrm{read} / 00359046265 \mathrm{a} 83 \mathrm{~b} 7 \mathrm{~b} 8273$

Gobierno del Perú (2016). Decreto supremo 004-2016-MC Reglamento de la Ley 29735. Recuperado de https://busquedas.elperuano.pe/normaslegales/decreto-supremo-que-aprueba-el-reglamento-de-la-ley-n-29735-decreto-supremo-n-004-2016-mc-1407753-5/

Gobierno Regional de Ucayali-DRE (2008). Proyecto educativo regional de Ucayali 2008-2021. Recuperado de http://repositorio.minedu. gob.pe/bitstream/handle/123456789/1063/626.\%20Proyecto\%20 
Educativo\%20Regional\%20de\%20Ucayali\%202008\%20-2021. pdf?sequence $=1$ \&isAllowed $=\mathrm{y}$

Hesse-Biber, S. N. y Leavy, P. (2011). The practice of qualitative research (2nd ed.). Los Angeles, CA: Sage.

Instituto Nacional de Estadística e Informática (2009). Censos Nacionales 2007: XI de población y VI de vivienda. Resumen Ejecutivo. Resultados definitivos de las comunidades indigenas. Recuperado de https://www.inei.gob.pe/media/ MenuRecursivo/publicaciones_digitales/Est/Lib0789Libro.pdf

Jiménez-Llanos, A. y Feliciano-García, L. (2006). Pensar el pensamiento del profesorado. Revista Española de Pedagogía, 64(233), 105-122. Recuperado de: https://www.jstor.org/stable/pdf/23765975.pdf?refreqid=excelsior\%3 A28c02a8c8acfb8a1f3bf1bf132e54868

Leiva, J. J. (2010). Educación intercultural y convivencia desde la perspectiva docente. Profesorado. Revista de Curriculum y Formación de Profesorado, 14(3), 251-274. Recuperado de https://recyt.fecyt.es/index.php/ profesorado/article/view/43407

León, E. (2014). Docencia, Interculturalidad y Educación Inicial. Prácticas docentes de Educación Intercultural Bilingüe en Educación Inicial en contextos andinos y amazónicos. Lima: Tarea.

López, L.E. (2001). La cuestión de la interculturalidad y la educación latinoamericana. ED-01/ PROMEDLAC VII/ Documento de Apoyo. Séptima Reunión del Comité Regional Intergubernamental del Proyecto Principal de Educación en América Latina y el Caribe. Unesco.

López-Vargas, B. y Basto-Torrado, S. (2010). Desde las teorías implícitas a la docencia como práctica reflexiva. Educación y Educadores, 13(2), 275-291. Recuperado de http://educacionyeducadores.unisabana.edu.co/index. php/eye/article/view/1699/2197

Martín, E., Pozo, J.I., Mateos, M., Martín, A. y Pérez Echeverría, M. del P. (2014). Infant, primary and secondary teachers' conceptions of learning and teaching, and their relation to educational variables. Revista Latinoamericana de Psicología, 46(1-3), 211-221. https://doi.org/10.1016/ S0120-0534(14)70024-X

Ministerio de Cultura (2016). Lista de pueblos indigenas. Recuperado de http:// bdpi.cultura.gob.pe/sites/default/files/Lista_completa_pueblos_indigenas/Listapueblosindígenasuoriginarios.pdf

Ministerio de Educación del Perú (1972). Política Nacional de Educación Bilingüe. Lima: Dirección Central de Publicaciones.

Ministerio de Educación del Perú (2008). Diseño Curricular Nacional de la Educación Básica Regular. Lima. 
Ministerio de Educación del Perú (2013). Documento Nacional de Lenguas Originarias del Perú. Lima: Minedu. Recuperado de http://www2.minedu. gob.pe/filesogecop/DNL-version\%20final\%20WEB.pdf

Ministerio de Educación del Perú (2013). Hacia una educación intercultural bilingüe de calidad. Propuesta Pedagógica. Lima: Minedu. Recuperado de: https://centroderecursos.cultura.pe/sites/default/files/rb/pdf/Hacia\%20 una $\% 20$ educacion $\% 20$ intercultural $\% 20$ bilingue $\% 20 \mathrm{de} \% 20$ calidad $\% 20$ Propuesta_pedagogica_EIB.pdf

Ministerio de Educación del Perú (2016). Programa Curricular de Educación Primaria. Recuperado de http://www.minedu.gob.pe/curriculo/pdf/programa-curricular-educacion-primaria.pdf

Ministerio de Educación del Perú (2016). Resolución ministerial 629-2016MINEDU: Plan Nacional de Educación Intercultural Bilingüe al 2021. Recuperado de http://www.grade.org.pe/forge/descargas/PLAN EIB.pdf

Ministerio de Educación del Perú (2016). Currículo Nacional. Lima: Minedu. Recuperado de http://www.minedu.gob.pe/curriculo/pdf/curriculonacional-de-la-educacion-basica.pdf

Organización Internacional del Trabajo (1989). Convenio sobre pueblos indígenas y tribales, 1989 (núm. 169). Parte I. Politica General, Artículo 5. Ginebra. Recuperado de https://www.ilo.org/dyn/normlex/es/f?p=NORMLEXPUB: 12100:0::NO:12100:P12100_INSTRUMENT_ID:312314:NO

Peschiera, R. (2010). Un análisis sobre la interpretación de los diferentes actores entorno a la educación intercultural y bilingüe y sus politicas. [Tesis de maestría]. Lima: Pontificia Universidad Católica del Perú, Escuela de Posgrado. Maestría en Políticas Educativas. Recuperado de http://tesis.pucp.edu. pe/repositorio/bitstream/handle/123456789/1370/PESCHIERA_RUJU_ ROMINA_ANALISIS.pdf?sequence $=1$ \&isAllowed $=\mathrm{y}$

Rodrigo, M.J., Rodriguez, A. y Marrero, J. (1993). Las teorías implícitas: una aproximación al conocimiento cotidiano. Madrid: Visor.

Ruiz, J.I. (1996). Metodología de la investigación cualitativa. Bilbao: Universidad de Deusto.

Simons, H. (2009). Case study research in practice. Londres: Sage. https://doi. org/10.4135/9781446268322

Tapia, J. y Cueto, S. (2017). El apoyo de FORGE al desarrollo del Currículo Nacional de la Educación Básica del Perú. Lima: Grade. Recuperado de http://www. grade.org.pe/forge/descargas/Sistematizaci\%C3\%B3n\%20Curriculo.pdf

Trumbull, D., Scarano, G. y Bonney, R. (2006). Relations among two teachers' practices and beliefs, conceptualizations of the nature of science, and their implementation of student independent inquiry projects. 
International Journal of Science Education, 28(14), 1717-1750. https://doi. org/10.1080/09500690600560928

Tubino, F. (2004). Interculturalidad para todos ¿̨un slogan más? Palestra: portal de asuntos públicos de la PUCP (pp. 1-6). Recuperado de http://repositorio. pucp.edu.pe/index/bitstream/handle/123456789/11898/interculturalidad_todos_Tubino.pdf?sequence $=1$ \&isAllowed $=y$

Tubino, F. (2005). Del interculturalismo funcional al interculturalismo crítico. Diálogos A: Culturas, Espiritualidades y Desarrollo Andino Amazónico, 1(1) Recuperado de http://red.pucp.edu.pe/wp-content/uploads/biblioteca/ inter_funcional.pdf

Tubino, F. (2015). La interculturalidad en cuestión. Lima: Fondo Editorial PUCP.

Tubino, F. y Zariquiey, R. (2004). Las prácticas discursivas sobre la interculturalidad en el Perú de hoy. Propuesta de lineamientos para su tratamiento en el sistema educativo peruano. Consultoría encargada por la Dirección Nacional de Educación Bilingüe Intercultural. Lima: Minedu. Recuperado de https://centroderecursos.cultura.pe/sites/default/files/rb/pdf/Las\%20 practicas $\% 20$ discursivas $\% 20$ sobre $\% 201$ a $\% 20$ interculturalidad $\% 20$ en\%20el\%20Peru\%20de\%20hoy.pdf

Valdiviezo, L. A. (2013). Cosmovisiones indígenas y construcciones sobre la interculturalidad en la educación bilingüe. Revista Peruana de Investigación Educativa, (5), 99-123. Recuperado de http://www.siep.org.pe/wp-content/uploads/282.pdf

Valdiviezo L. A. y Valdiviezo Arista, L. M. (2008). Política y práctica de la interculturalidad en la educación peruana: análisis y propuesta. Revista Iberoamericana de Educacion, (45), 1-12. Recuperado de http://repositorio.minedu.gob.pe/bitstream/handle/123456789/901/531.\%20 Pol\%C3\%ADtica\%20y\%20pr\%C3\%A1ctica\%20de\%20la\%20interculturalidad $\% 20$ en $\% 201$ a $\% 20$ educaci $\%$ C3\%B3n $\% 20$ peruana $\% 20$ an\%C3\%A1lisis\%20y\%20propuesta.pdf?sequence=1\&isAllowed $=y$

Walsh, C. (2000). Propuesta para el tratamiento de la Interculturalidad en la Educación. Lima: Minedu. Recuperado de https://centroderecursos. cultura.pe/sites/default/files/rb/pdf/Propuesta\%20para\%20el\%20tratamiento\%20de $\% 201 \mathrm{l} \% 20$ interculturalidad $\% 20$ en $\% 201 \mathrm{a} \% 20$ educacion. pdf

Walsh, C. (2009). Interculturalidad crítica y educación intercultural. En J. Viaña, L. Tapia y C. Walsh (eds.), Construyendo Interculturalidad Critica (pp. 75-96). Bolivia: Instituto Internacional de Integración del Convenio Andrés Bello III - CAB. 
Zavala, V., Cuenca, R. y Córdova, G. (2005). Hacia la construcción de un proceso educativo intercultural: elementos para el debate. Lima: Ministerio de Educación-Dinfocad-Proeduca-GTZ.

Zúñiga, M. y Ansión, J. (1997). ¿Qué entender por interculturalidad? En Interculturalidad y educación en el Perú (pp. 9-20). Lima: Foro Educativo,. Recuperado de http://red.pucp.edu.pe/ridei/wp-content/uploads/biblioteca/inter59.PDF 